\title{
Sea quark polarization and semi-inclusive DIS data.
}

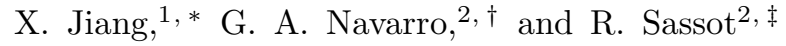 \\ ${ }^{1}$ Department of Physics and Astronomy, Rutgers, the State University of New Jersey \\ 136 Frelinghuysen Road, Piscataway, NJ 08854 USA. \\ ${ }^{2}$ Departamento de Física, Universidad de Buenos Aires \\ Ciudad Universitaria, Pab.1 (1428) Buenos Aires, Argentina
}

\begin{abstract}
We investigate the potential impact of forthcoming Jefferson Lab semi-inclusive polarized deep inelastic scattering proton measurements in the determination of the sea quark polarization in the nucleon by means of a next to leading order global QCD analysis. Specifically, we estimate the resulting improvement in the constraints on polarized parton densities for the different flavors, which is found to be significant for up and strange quarks, and the correlation between remaining uncertainty ranges for each of the parton species.
\end{abstract}

PACS numbers: 12.38.Bx, 13.85.Ni

Keywords: Semi-Inclusive DIS; perturbative QCD.

\section{INTRODUCTION}

The way in which sea quarks are polarized inside nucleons has been a persisting question ever since the spin structure of the proton began to be unveiled by polarized DIS experiments and even today, in spite of several successful experimental programs, remains to a large extent unanswered. Contrary to the common belief before the paradigmatic EMC experiment at the end of 1980s [1, 2], the data obtained by the collaboration suggested that sea quarks and gluons in the nucleon carried non negligible polarization. However this conclusion was, and has been for many years, conditional upon rather strong assumptions on isospin symmetry extended to polarized phenomena. In the subsequent years, isospin symmetry itself become seriously questioned [3] and consequently the sea quark polarization turned into an even more elusive question.

Global QCD analyses including semi-inclusive measurements of polarized lepton-nucleon deep inelastic processes began to change this situation more recently [4, [5], and today these data allow to constrain the extraction of polarized parton densities in QCD global fits [ [] . The effectiveness of these constraints of course relies on the precision of the data and this is why the forthcoming generation of semi-inclusive experiments is crucial.

In a QCD global fit the uncertainty range of the resulting parton densities can be estimated by analyzing the profile of the $\chi^{2}$-function of the fit to data against variations in the different features of the densities. This technique has been widely used in extractions of unpolarized parton densities 7$]$ and more recently has been implemented in the polarized case [6] providing reliable constraints on their different features, such as the net polarization carried by each parton flavor.

Of course, the values each flavor polarization, or some other parameter, may take within these constraints are not independent, but become correlated. Even in the case where there are enough independent observables to extract in principle all the parton densities, the uncertainties in the measurements of those observables, together with the theoretical uncertainties inherent in the fitting procedure, conspire against the independence of parton densities and results in correlations. A strong correlation between two parton densities, consequently means that neither of them is actually well determined. The inclusion of new and more precise data should not only reduce the uncertainty ranges for each flavor but also those correlations.

In this article we investigate the impact of the inclusion of a series of semi-inclusive deep inelastic scattering measurements to be performed at Jefferson Lab [8] in a next to leading order (NLO) QCD global fit to all the available inclusive and semi-inclusive deep inelastic scattering data. In order to do this we take into account the kinematical coverage, statistics and level of uncertainty expected for the measurements. We also analyze the correlation between the uncertainty ranges of the different sea quark polarizations. As result of this analysis we find that the forthcoming Jefferson Lab experiment will effectively contribute to constrain the sea quark polarization in the proton. The most significant improvement is found in up sea quark distributions, and also with a noticeable effect for strange quarks.

\footnotetext{
*Electronic address: jiang@jlab.org

†Electronic address: gabin@df.uba.ar

‡Electronic address: sassot@df.uba.ar
} 
TABLE I: Inclusive and semi-inclusive data used in the fit.

\begin{tabular}{|c|c|c|c|c|}
\hline Collaboration & Target & Final state & \# points & Refs. \\
\hline EMC & proton & inclusive & 10 & {$[2]$} \\
\hline SMC & proton, deuteron & inclusive & 12,12 & {$[13]$} \\
\hline E-143 & proton, deuteron & inclusive & 82,82 & {$[14]$} \\
\hline E-155 & proton, deuteron & inclusive & 24,24 & {$[15]$} \\
\hline Hermes & proton, deuteron,helium & inclusive & $9,9,9$ & [20] \\
\hline E-142 & helium & inclusive & 8 & {$[16]$} \\
\hline E-154 & helium & inclusive & 17 & {$[14]$} \\
\hline Hall A & helium & inclusive & 3 & [18] \\
\hline COMPASS & deuteron & inclusive & 12 & [21] \\
\hline $\mathrm{SMC}$ & proton, deuteron & $h^{+}, h^{-}$ & 24,24 & [19] \\
\hline \multirow[t]{2}{*}{ Hermes } & proton, deuteron, helium & $h^{+}, h^{-}, \pi^{+}, \pi^{-}, K^{+}, K^{-}, K^{T}$ & $36,63,18$ & {$[20]$} \\
\hline & Total & & 478 & \\
\hline
\end{tabular}

The improvement in both distributions will be related to the inclusion in the fit of more precise data on charged meson electroproduction. Another interesting effect of the inclusion of these data is the softening of the correlation between the allowed range for the polarization of up and down sea quarks.

In the following section, we settle definitions and conventions for the global fitting procedure and the way in which we study the profile of the $\chi^{2}$ function, we discuss the characteristics of the forthcoming semi-inclusive experiment, and explain how we evaluate the impact of it in a global fit. Then, we compare the results coming from the analysis of the set of data available at present, against those that would come from the data set enlarged with the forthcoming measurements, both for the individual uncertainty ranges, for the net polarization of the different flavors, and for the correlations between flavors. Finally, we present our conclusions.

\section{GLOBAL QCD FITS AND NEW DATA}

In the present analysis we implement the NLO QCD global fit to existing data along the lines of what was done in reference [6] but restricting the input fragmentation functions to those of reference [9], which were shown to give the best fits to combined polarized data. The NLO expressions for both inclusive and semi-inclusive spin-dependent asymmetries and evolution equations for the parton densities can be found in [10, 11] and [12] respectively.

The data sets analyzed include only points with $Q^{2}>1 \mathrm{GeV}^{2}$, listed in Table $\llbracket$ and totaling 137 , 139 , and 37 points, from proton, deuteron, and helium targets respectively, from polarized inclusive deep inelastic scattering plus 60,87 , and 18, from proton, deuteron, and helium targets respectively from semi-inclusive deep inelastic scattering.

The main conclusions reached in reference [6] were that using the Lagrange multiplier approach [22] as a mean to explore the profile of the $\chi^{2}$ function against different degrees of polarization in each parton flavor, definite estimates for the uncertainty in the net polarization of each flavor, and in the parameters of the polarized parton distributions, can be obtained. The overall result is a well constrained scenario where semi-inclusive data is not only consistent with inclusive measurements, but improves the constraining power of the fit for all the distributions, being crucial for the light sea quarks.

The best fits suggest an overall picture for the quark densities at $10 \mathrm{GeV}^{2}$ where, within uncertainties, up quarks are almost $100 \%$ polarized parallel to the proton, down quarks anti-parallel in a similar proportion, and sea quarks have a small and flavor symmetric negative polarization. The first moment of the gluon distribution is found to be in agreement with the most recent direct measurements [23] close to 0.6 , constrained to be smaller than 0.8 and larger than -0.05 within a conservative increase in the $\chi^{2}$ value within a two percent range $\left(\Delta \chi^{2}=2 \%\right)$.

In order to evaluate the impact of the forthcoming semi-inclusive proton data of Jefferson Lab, we included in the global analysis the expected values of the $\pi^{+}, \pi^{-}, K^{+}$and $K^{-}$semi-inclusive asymmetries on a polarized proton target, computed with the best set of parton densities obtained in [ 6 ] with expected experimental uncertainties, as an additional set of points to be fitted. The projected statistical accuracies of these asymmetries are based on a total of 225 hours of $6 \mathrm{GeV}$ polarized electron beam on a polarized $\mathrm{NH}_{3}$ target. The electron beam current is assumed to be $80 \mathrm{nA}$ with a polarization of $80 \%$. The standard Jefferson Lab Hall C polarized $\mathrm{NH}_{3}$ target of $3 \mathrm{~cm}$ thickness and 80 $\%$ polarization is assumed. The scattered electron will be detected at 30 degree with an array of lead-glass detectors in conjunction with a threshold gas Cherenkov counter, covering a solid angle of 210 msr. The produced hadron will 
be detected in coincidence using the standard Hall C High Momentum Spectrometer (HMS) at 10.8 degree and a central momentum of $2.7 \mathrm{GeV} / \mathrm{c}\left(z_{\pi} \approx 0.5\right)$. The HMS spectrometer has a solid angle of 6 msr and a momentum acceptance of $\pm 10 \%$.

With this enlarged set of asymmetries to be fitted, we have redone the analysis of [6] adding a detailed study of the correlations, and compared the resulting constraints on polarization with those of the original fit.

\section{RESULTS}

We start with the estimates for the uncertainties in the polarization of the different quark flavors. In Figure 1 we show the outcome of varying the $\chi^{2}$ of the NLO fits with the set of data available at present, in the following referred to as "standard fit", and the "improved fit", which includes the asymmetries expected to be measured by the E04-113, against the first moment of the respective polarized parton densities $\delta \bar{q}$ at $Q^{2}=10 \mathrm{GeV}^{2}$, one at a time. This is, to minimize

$$
\Phi\left(\lambda_{q}, a_{j}\right)=\chi^{2}\left(a_{j}\right)+\lambda_{q} \delta q\left(a_{j}\right) \quad q=u, \bar{u}, d, \bar{d}, s, g .
$$

where $\lambda_{q}$ is the Lagrange multiplier associated to the polarization of a given quark flavor $q, a_{j}$ are the parameters to be fitted, and the $\chi^{2}$ definition is the most simple and commonly used in fits to polarized data, namely,

$$
\chi^{2}=\sum_{i=1}^{N} \frac{\left(T_{i}\left(a_{j}\right)-E_{i}\right)^{2}}{\sigma_{i}^{2}} .
$$

In Equation (2), $E_{i}$ is the measured value of a given observable, $T_{i}$ is the corresponding theoretical estimate computed with a given set of parameters for the polarized parton densities, and $\sigma_{i}$ is the error associated with the measurement,

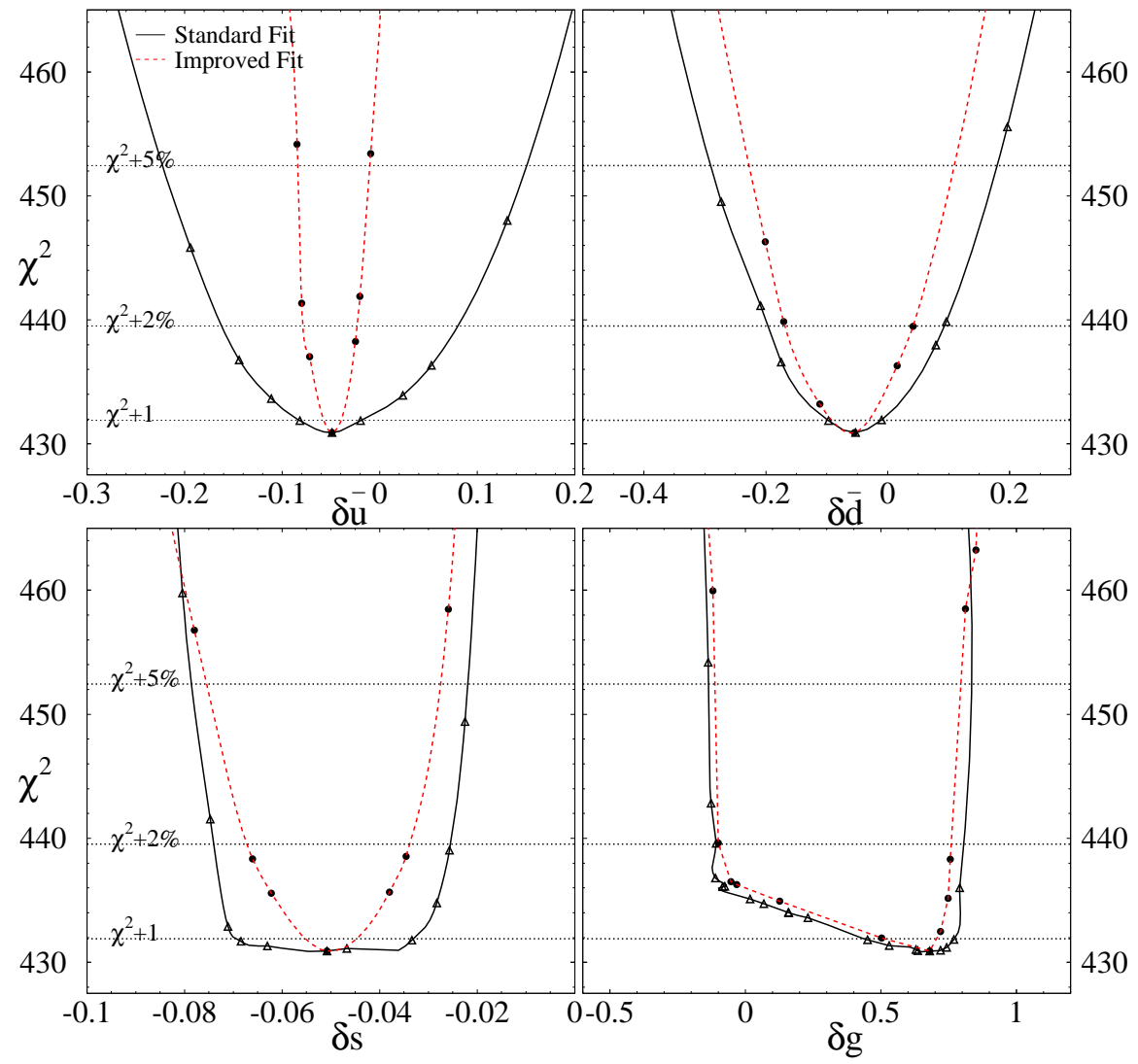

FIG. 1: Profile of the $\chi^{2}$ function against parton polarizations. 


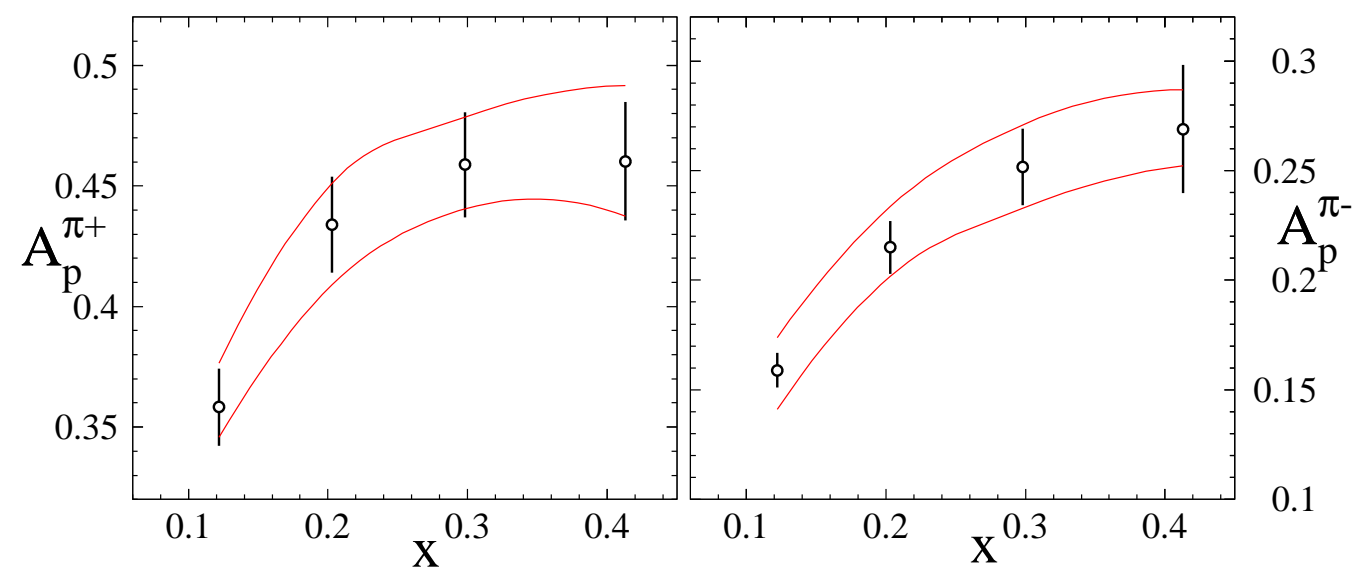

FIG. 2: Uncertainty bands for $A_{p}^{\pi+}$ and $A_{p}^{\pi-}$ asymmetries coming the "improved fit" and E04-113 expected uncertainties.

usually taken to be the addition of the reported statistical and systematic errors in quadrature. Notice that the additional set of asymmetries included does not contribute to $\chi^{2}$ when it is computed with parton densities corresponding to the best fit of reference [ $]$, the same densities used to generate the asymmetries, situation that happens at the minima of the curves. As the distributions change in order to increase or reduce the polarization of a given flavor, the $\chi^{2}$ obtained with one or another set begin to differ. The solid lines in Figure 1 correspond to the analysis of the standard set of data, while the dashed lines includes the estimated impact of future measurements.

As expected, the most noticeable effect is in $\bar{u}$ polarization due to the maximal sensitivity of the semi-inclusive asymmetries on proton targets to this distribution, as discussed in [5]. For the $\bar{d}$ and $\bar{s}$ distributions, the impact of the future measurements is comparatively suppressed by the weakness of the electric charge factor associated with these flavors, however there is a noticeable improvement for the $\bar{s}$ quark uncertainty near the minimum. In the previous analysis this distribution was mainly constrained by positivity resulting in flat $\chi^{2}$ distribution around the minimum but here shows a nice parabolic profile. Notice that both in the analysis of reference [5] and in the present one, we are forced to assume $\Delta \bar{s}=\Delta s$ since there is not enough data yet to discern alternatives. This assumption implies a strong constraint on $\delta \bar{s}$ even though the measured asymmetries are less sensitive to this distribution than to $\delta \bar{u}$ and $\delta \bar{d}$. On the contrary, the relation between $\delta \bar{u}$ and $\delta u$ and the same for $\delta \bar{d}$ and $\delta d$ comes from the fit. The impact on the gluon distribution is not significant and mainly indirect, coming as found in reference [5] through the constraints on the sea quark distributions, which are now better defined. It is worth mentioning that the impact of the kaon data is very mild, being mostly the pion asymmetries responsible for the changes.

As discussed in references [5, 7], in modern extractions of parton densities it is customary to consider alternative sets of parton densities within an increase between $2 \%$ and $5 \%$ in $\chi^{2}$, as a conservative estimate for the range of uncertainty of the global fit. In order to estimate the corresponding uncertainty range in the computation of a given observable, it is customary to take it as the range of variation of the observable within the alternative sets. This is precisely what we show in Figure 2 where we plot the uncertainty bands of $A_{p}^{\pi+}$ and $A_{p}^{\pi-}$ corresponding to $\Delta \chi^{2}=5 \%$ in the improved fits as the area between the dashed lines. For comparison we include in the plots the values for these pion asymmetries at the kinematics of the forthcoming Jefferson Lab experiment, computed with the set of [6] and which where included as "data" in the "improved fit", together with the expected error bars. In this way, we can see not only the consistency of the results but also the appropriateness of the choice of $\Delta \chi^{2}=5 \%$ Similar uncertainty bands obtained for $\Delta \chi^{2}=5 \%$ but in the "standard fit" were found to be twice as large in 6 .

As it was pointed out in the introduction, an important feature to keep in mind regarding the range of variation of the polarization of the different flavors is that they are correlated. For example, they cannot be expected to hold simultaneously; in consequence, for a given allowed range in $\chi^{2}$, two or more flavors may cannot take their respective maximum departures from the best fit value together. For this reason it is worth while to study such correlations between the different flavors, and how these correlations change with the inclusion of additional data. This can be done systematically generalizing Equation (1) for more than one flavor polarization, with independent Lagrange multipliers, scanning the profile of the $\chi^{2}$ function in the range of variation of them. 

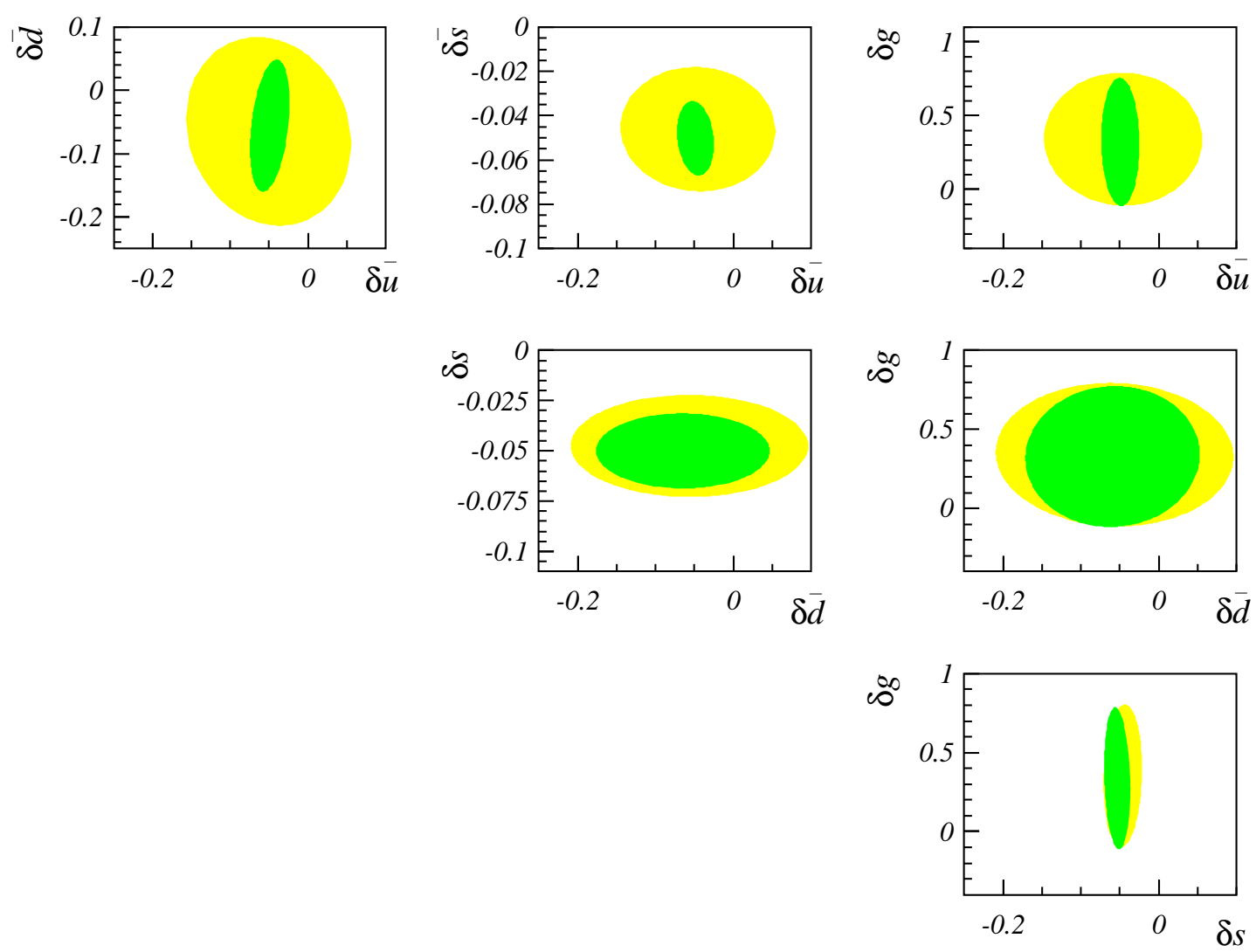

FIG. 3: Correlations between the flavor polarizations within a $5 \%$ increase in $\chi^{2}$ in both the standard and the improved fits.

In Figure 3 we show the allowed range of polarization within a $5 \%$ increase in $\chi^{2}$ in the $\delta \bar{u}-\delta \bar{d}, \delta \bar{u}-\delta \bar{s}, \delta \bar{u}-\delta g$, $\delta \bar{d}-\delta \bar{s}, \delta \bar{d}-\delta g$, and $\delta \bar{s}-\delta g$ planes. In order to simplify the plots we have approximated the actual contours by ellipses, the darker ones obtained with the "improved fit", while lighter being those coming from the "standard fit". Again the most prominent effect is the shrinkage of the $\delta \bar{u}$ and $\delta \bar{s}$ uncertainty range.

In these plots the correlations between the polarization of the different flavors are represented by the angles between the axes of the ellipses and the coordinated axes. A positive or negative $\pi / 4$ difference would imply a maximal positive or negative correlation respectively, and that both polarizations are weakly constrained. This is the case, for example for $\delta \bar{u}$ and $\delta \bar{d}$ in the "standard fit", situation that is corrected in the improved version. In the remaining cases, the axes of the ellipses are almost parallel to those of the coordinates suggesting mild correlations between the different pairs of flavors.

There is however, a remaining subtle correlation between $\delta \bar{u}$ and $\delta \bar{d}$ and between $\delta \bar{u}$ and $\delta \bar{s}$, that the enlarged set of asymmetries included in the fit is still not able to remove. In the first case the residual correlation is positive, while in the latter it is negative. The gluon polarization also seams to have negligible correlation with that of the anti-quarks, with only a very slight positive tendency with $\delta \bar{s}$ in the standard fit, which is removed in the improved one.

Another interesting correlation to investigate is the one between the total polarization carried by anti-quarks and that of the gluons. This in practice amounts to associate a Lagrange multiplier in Equation (1) to the sum over the anti-quark species $\delta \Sigma_{\bar{q}}=(\delta \bar{u}+\delta \bar{d}+\delta \bar{s})$ and another to the gluon polarization. In Figure 4 we see that there is no significant correlation between $\delta \Sigma_{\bar{q}}$ and $\delta g$. Notice also how significantly the asymmetries expected to be measured by E04-113 will help to constrain the anti-quark polarization. 


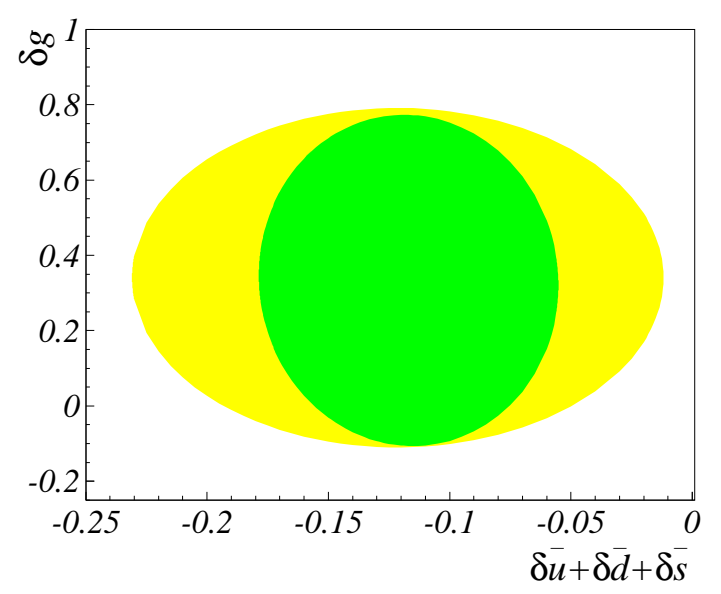

FIG. 4: Correlation between $\delta \Sigma_{\bar{q}}$ and $\delta g$.

\section{CONCLUSIONS}

We have analyzed the potential impact of forthcoming semi-inclusive polarized deep inelastic scattering proton measurements in the determination of sea quark polarization in the nucleon by means of a next to leading order global QCD analysis. We find that the inclusion of this data will effectively contribute to constrain the sea quark polarization in the proton. The most significant improvement is found in the up anti-quark distribution, and with an also noticeable effect for strange anti-quarks. For down anti-quarks, the new data will have a smaller but nonnegligible effect. Regarding the correlations, we found that the forthcoming data will reduce the apparent correlation found between $\delta \bar{u}$ and $\delta \bar{d}$ in the standard fit of reference [6] leading to a picture where the sea quark densities and their uncertainties can be determine independently.

\section{ACKNOWLEDGEMENTS}

We warmly acknowledge Daniel de Florian for comments and suggestions. R.S. is grateful to Jefferson LAB for the hospitality during his visit where this analysis was completed. This work was partially supported by CONICET, Fundación Antorchas, UBACYT and ANPCyT, Argentina and the US National Science Foundation grant PHY 03-54871.

[1] J. Ashman et al. [European Muon Collaboration], Phys. Lett. B 206, 364 (1988).

[2] J. Ashman et al. [European Muon Collaboration], Nucl. Phys. B 328, 1 (1989).

[3] S. Kumano, Phys. Rept. 303, 183 (1998).

[4] D. de Florian, O. A. Sampayo and R. Sassot, Phys. Rev. D 57, 5803 (1998).

[5] D. de Florian and R. Sassot, Phys. Rev. D 62, 094025 (2000).

[6] D. de Florian, G. A. Navarro and R. Sassot, Phys. Rev. D 71, 094018 (2005).

[7] A.D.Martin, R.G.Roberts, W.J.Stirling and R.S.Thorne, Eur.Phys.J.C28, 455 (2003); J.Pumplin, D.R.Stump, J.Huston, H.L.Lai, P.Nadolsky and W.K.Tung, J.High Energy Phys. 07 (2002) 012.

[8] X. Jiang et al. Jefferson Lab experiment E04-113: Semi-Inclusive Spin Asymmetries on the Nucleon Experiment (hep-ex/0412010).

[9] S. Kretzer, Phys. Rev. D 62 (2000) 054001.

[10] R. Mertig, W. L. van Neerven, Z.Phys.C70, 637,(1996); W. Vogelsang, Phys.Rev.D54, 2023, (1996).

[11] M. Gluck, E. Reya, M. Stratmann and W. Vogelsang, Phys.Rev.D53, 4775 (1996).

[12] D. de Florian, C.A. Garcia Canal, and R. Sassot, Nucl. Phys. B470, 195 (1996).

[13] SMC Collaboration, B. Adeva et al., Phys. Rev. D58, 112001 (1998).

[14] E143 Collaboration, K. Abe et al, Phys. Rev. D 58, 112003 (1998).

[15] E155 Collaboration, P. L. Anthony, et al., Phys. Lett. B463, 339 (1999); G. S. Mitchell, Ph.D. Thesis University of Wisconsin-Madison, SLAC-Report-540 (1999) 
[16] E142 Collaboration, P. L. Anthony, et al., Phys. Rev. D 54, 6620 (1996).

[17] E154 Collaboration, K. Abe et al., Phys. Rev. Lett. 79, 26 (1997); Phys. Lett. B405, 180 (1997).

[18] HALL A Collaboration X. Zheng, et al., nucl-ex/0405006

[19] B. Adeva et al., SMC Collab., Phys. Lett. B369, 93 (1996), ibid. 420180 (1998).

[20] HERMES Collab., A. Airapetian, et al. hep-ex/0407032 K. Ackerstaff et al,, Phys. Lett. B464, 123 (1999).

[21] A. Bressan, for the COMPASS Collaboration, hep-ex/0501040

[22] J. Pumplin, D. R. Stump and W. K. Tung, Phys. Rev. D 65, 014011 (2002)

D. Stump et al., Phys. Rev. D 65, 014012 (2002)

[23] J. R. Ellis and M. Karliner, arXiv:hep-ph/0501115 